\title{
OPINIÓN PÚBLICA Y SALUD: LAS DISCUSIONES SOBRE EL DESARROLLO DE LOS SERVICIOS DE SALUD PÚBLICA EN EL SALVADOR 1948-1957
}

\section{Public Opinion andHealth: Discussions regarding the Development of Public Health Services in El Salvador 1948-1957}

\author{
Herberth Stanley Morales-Herrera*
}

Resumen: El artículo pretende ser un primer acercamiento a la discusión que se realizó en torno a los servicios de la salud pública en El Salvador entre 1948-1957. Metodológicamente se realiza un análisis del discurso médico de una manera diacrónica. Para ello se toma en cuenta los aportes de Michel Foucault, quien prestó atención al discurso médico por medio de la relación entre la salud pública y el Estado nacional. Tres tipos de fuentes se trabajan: periódicos estudiantiles, revistas y documentos de instituciones Estatales. Se concluye que los médicos salvadoreños articularon una narrativa que influyó en instancias académicas y estatales.

Palabras clave: análisis del discurso, salud pública, opinión pública.

Abstract: The article is an initial survey of discussions held in El Salvador from 1948 to 1957 regarding public health services. Methodologically, we analyzed medical discourse diachronically. The contributions of Michel Foucault informed our survey, given that he studied medical discourse by examining the relationship between public health and the national state. We examined three types of sources: student newspapers, magazines, and documents of state institutions. We conclude that Salvadoran doctors articulated a narrative that influenced academic and state institutions.

Keywords: discourse analysis, public health, public opinion.

\footnotetext{
* Herberth Stanley Morales Herrera. Licenciado en Historia por la Universidad de El Salvador, El Salvador. Coordinador de la Escuela de Formación Política y Ciudadana en la Universidad Centroamericana "José Simeón Cañas", El Salvador. Temas de especialización: histórica local e historia social de la salud pública. Correo electrónico: hmorales@ uca.edu.sv. ORCID: https://orcid.org/0000-0001-8973-0621.
}

Enviado a dictamen: 24 de abril de 2018 Aprobación: 25 de septiembre de 2018 Revisiones: 2 


\section{Contexto historiográfico}

L a historia de la salud en El Salvador aún tiene pendiente la tarea de consolidar una trayectoria, como lo han hecho ya la historia política y económica. A pesar de haberse realizado algunos esfuerzos académicos (Juárez, 2015; Durán, 2016), el tema de la salud pública como problema histórico ha dado pocos pasos después de haber dejado atrás su punto de salida. ${ }^{1}$ En esta dirección, pueden citarse algunas publicaciones recientes del Ministerio de Salud: Mortalidad y años de vida potencialmente perdidos por enfermedadesno transmisibles en El Salvador 2011-2015 (Zelaya y Mejía, 2018) y Calidad microbiológica de agua envasada en El Salvador 2014-2015 (Quinteros y Mejía, 2018).

Asimismo, pueden mencionarse las obras del oftalmólogo Carlos Infante Meyer, quien como profesional de la salud abordó algunos elementos de la historia de la salud pública del país. Por ejemplo, en su libro titulado Historia de la medicina en El Salvador se basa en una gran cantidad de fuentes y recopila datos que bien podrían ser interpretados desde la óptica propia del historiador (Infante, 2000). Además, destacan los trabajos de algunos historiadores como López Bernal y Roberto Turcios, quienes contribuyeron a contextualizar el tema de la salud pública sin ser éste el eje principal de sus trabajos (López, 2000; Turcios, 2003).

Por otra parte, la tesis de Mario Daniel Ernesto Oliva Mancia, titulada Ciudadanía e higienismo social en El Salvador 1880-1932, es una novedad para el contexto historiográfico salvadoreño. En esta obra, el autor hace un análisis desde la historia de las ideas articulando elementos de salubridad, violencia y formación de la ciudadanía. En este trabajo, a través de la categoría de "higienismo social", el autor invita a reflexionar sobre los discursos y mecanismos de control social que usó el Estado salvadoreño, cuyo objetivo era justificar un modelo de desarrollo anclado en la modernidad liberal (Oliva, 2011).

Hay que destacar que en algunas de las tesis de medicina que se encuentran en poder de la Universidad de El Salvador se abordan elementos de la historia de la salud que muy bien pueden ayudar al análisis. Por ejemplo, el Dr. Carlos Alberto Villatoro, en su tesis titulada Breve estudio de la medicina rural en El Salvador, reflexionó acerca del sistema de salud salvadoreño tomando como base la década de 1960 (Villatoro, 1968).

Si a lo anterior agregamos la variable de la opinión pública ${ }^{2}$ para reflexionar sobre el tema de la salud, el panorama se vuelve más complejo. El presente esfuerzo se enmarca en este reto.

\section{Planteamientos desde un gremio de médicos: la revista Archivos del Colegio Médico de El Salvador}

Con un panorama historiográfico como el anterior en el tema de la salud pública, la tarea de investigar se torna difícil, como salir de cacería en búsqueda de fuentes primarias y bibliográficas, que son dispersas ya que no existe una tradición historiográfica de referente nacional. Parecida preocupación contempló el médico salvadoreño Roberto J. Calderón en 1949, al no encontrar un centro académico que le brindara bibliografía del país relativa a la investigación que estaba realizando sobre lesiones cardíacas, la cual publicó en junio de ese año en la entonces novel revista Archivos del Colegio Médico de El Salvador (Calderón, 1949: 73-74).

La referida revista apenas había comenzado a publicar en 1948 sus primeros números, y fue en la siguiente década cuando se volvió una referencia informativa para la ciudadanía interesada en el tema de la salud pública en El Salvador. Estoúltimo constituye el punto de interés del presente artículo, donde se analizan las publicaciones de esta revista, así como otras en las que se examinó la realidad de la salud pública nacional, bajo las siguientes dos preguntas: ¿cuáles fueron los temas y argumentos médicos que se vertieron a la opinión pública?, iquiénes fueron los interlocutores de los médicos en la discusión sobre el desarrollo de los servicios de salud pública en El Salvador durante la década de 1950 ?

La revista Archivos del Colegio Médico de El Salvador, creada por el Colegio Médico de El Salvador en el año indicado - 1948 - , se convirtió en la herramienta de difusión de los galenos que opinaban sobre la 
calidad de los servicios de salud pública y sobre sus investigaciones médicas, que tenían principalmente fundamento empírico en el trabajo diario del hospital Rosales, el más importante en el país. En este sentido, en el siglo XX no era una novedad que los hospitales fueran instancias generadoras de conocimiento. Para Foucault (1978), el proceso de medicalización que se llevó a cabo en los hospitales hacia mediados del siglo XVIII en Occidente generó en estas instancias espacios destinados a curar y también a producir conocimiento médico sobre las enfermedades. Él mismo agrega que antes del siglo XVIII los hospitales eran lugares de confinación para los enfermos en sus últimos días de vida, donde se preparaban para salvar sus almas. En síntesis, para Foucault el hospital dejó de ser una común infraestructura arquitectónica para moribundos y pasó a convertirse en un hecho médico sujeto a la observación de los galenos.

El Colegio Médico de El Salvador, uno de los actores más importantes que participaban en la discusión sobre salud pública, se institucionalizó en 1944, y a partir de entonces organizó los congresos nacionales de medicina (Colegio Médico de El Salvador, 1949b: 268). Principalmente por estos motivos, y por la revista que editaba, este colegio se convirtió en un centro de opinión importante en cuanto a la discusión sobre salud pública entre 1948 y 1957 en el país.

Pero antes de entrar de lleno a los argumentos médicos, es necesario retomar de nuevo a Michel Foucault (2004), quien sugiere prestar atención a la creación de las instituciones vinculadas con la salud, y particularmente al rol discursivo de los médicos, quienes van influyendo en el espacio social. En definitiva, Foucault argumenta que es la mirada de los médicos y su actuar como representantes de las instituciones sanitarias la que va formando una articulación para llevar a cabo una vigilancia constante en las sociedades, es decir, la activa influencia del conocimiento médico se hace presente en la cotidianidad del ciudadano de la modernidad. A este proceso Foucault lo llama la "medicalización rigurosa, militante y dogmática de la sociedad", según el cual los médicos se vuelven "un clero de la terapéutica" (Foucault, 2004: 57) a quienes consultar sobre la salud del cuerpo social. Mucho de esto puede notarse en las opiniones de los médicos o generadores de opinión que se citarán a continuación.

Una de las primeras ideas que plantearon los médicos en 1949, a la luz de la "medicina social", ${ }^{3}$ fue la de concebir la salud de una manera "tan importante como el propio derecho a la libertad personal y a la educación" (Colegio Médico de El Salvador, 1949a: 133). En otras palabras, se consideraba la salud un tema político, de la misma manera que un liberal estimaría la libertad. Esta aseveración fue expuesta por una comisión designada por el Colegio Médico de El Salvador que asesoró a los legisladores en la elaboración del anteproyecto de la constitución de 1950. El eco de esta primera idea de los médicos se deja ver en el concepto de Estado y de la forma de gobierno presente en el artículo 2 de la Constitución salvadoreña de 1950: "Es obligación del Estado asegurar a los habitantes de la república el goce de la libertad, la salud, la cultura, el bienestar económico y la justicia social" (CDJ, 1950: 2). Usualmente este artículo se interpreta como un cambio en la concepción filosófica del derecho salvadoreño y no como parte de un cuerpo de ideas también presentes en otros gremios de profesionales como podía ser el de los médicos (Turcios, 1993: 31).

En relación con lo anterior, deben tomarse en cuenta los cambios políticos iniciados en El Salvador tras el "Golpe de los Mayores"4 del 14 de diciembre de 1948, que abrió la idea de considerar al Estado como un actor protagónico en la economía y en las áreas de inversión social (Turcios, 2003: 20-86), aunque debe decirse que esta tendencia se esbozó en la década de 1930. Uno de los quiebres históricos fue dejar a un lado la Constitución de 1886, de concepción liberal, por la de 1950, que tenía principios acordes a las posturas de un Estado intervencionista (Turcios, 1993: 31), como lo promulgó la Comisión Económica para América Latina y el Caribe (CEPAL) (Rojas, 1993: 106). Eran tiempos de modernización del Estado y de la economía salvadoreña, sin dejar de lado el autoritarismo.

Una segunda idea que plantearon los médicos desde la revista fue la de considerar las condiciones de ruralidad del país. En el número de diciembre de 1949 aparece un 
artículo del Dr. José Pacheco, quien era médico auxiliar en la División de Epidemiología adscrita a la Dirección General de Sanidad (DGS), que fue el antecedente del Ministerio de Salud en El Salvador. Pacheco, influido por su experiencia en México, planteó la necesidad de estructurar un sistema de servicios médicos rurales, adecuando los aspectos técnicos al medio, con la misión de prevenir y curar las enfermedades en un país con una estructura demográfica mayoritariamente rural (Pacheco, 1949: 219-242). Al describir los centros asistenciales rurales del país, expresó lo siguiente: "Sus actividades se concretan al ejercicio de la medicina curativa descuidando por completo las labores de la medicina preventiva e higiénica. Allí radica uno de los mayores desaciertos" (Pacheco, 1949: 223).

A propósito, el interés por la medicina preventiva se tornó uno de los puntos centrales de la discusión durante el periodo en cuestión. Por ejemplo, el Colegio Médico en su editorial de marzo de 1950 destacaba la importancia de que la DGS estuviera dirigida por especialistas en medicina. Sin embargo, criticó que en materia de medicina preventiva los centros asistenciales no se encontraran bajo la supervisión técnica de la DGS, y mencionó que se esperaba que esa situación se corrigiera en el corto plazo (Colegio Médico de El Salvador, 1950: 51). La medicina preventiva durante ese periodo en el país tenía cuatro características y se centraba fundamentalmente en: identificar las principales causas de muerte, incidir en los factores ambientales vinculados a la morbilidad, realizar campañas nacionales para la prevención de enfermedades y definir la zona rural como la más necesitada de asistencia médica.

Este interés por la medicina preventiva se produjo a raíz del tipo de enfermedades que estaban configurando el cuadro de las principales causas de muerte en el país. Valga citar que, entre 1950 y 1957, la gastroenteritis y el paludismo fueron las dos primeras causas de muerte, las cuales son esencialmente prevenibles si se intervienen las condiciones sanitarias del ambiente (ver Cuadro l). Incluso, en 1949 el Dr. Juan Allwood Paredes, director de la DGS, afirmaba que se le prestaba poco interés al estudio de los padecimientos gastrointestinales a pesar de la influencia que tenía en los índices de mortalidad (Allwood, 1949: 106). Además, en 1956, en un estudio de la División de Epidemiología de la DGS publicado en la revista Archivos del Colegio Médico de El Salvador, se sostenía que, pese a los avances alcanzados en los últimos años para prevenir el paludismo, esta enfermedad seguía causando muertes en el país de manera significativa (Peñalver et al,. 1956: 1).

Los médicos que escribían en la revista sostenían, asimismo, que los profesionales de la salud se concentraban principalmente en la capital salvadoreña. Por ejemplo, en 1949 El Salvador contaba con 257 médicos, de los cuales 146 estaban en la capital (Grimmer, 1950: 214). Cuatro años después, en 1953, se contabilizaron 319 médicos que ejercían la profesión, y, de éstos, 183 laboraban en la capital (Infante, 2000: 319). Lo dicho anteriormente le brinda sentido a la siguiente exhortación que hizo el Colegio Médico de El Salvador en relación con el ejercicio de la medicina como profesión:

El médico de consulta externa debe procurar no faltar por causas baladíes porque lo necesitan enfermos pobres con largas horas de espera quienes se han trasladado (muchos de ellos) desde lugares distantes tal vez a pie o haciendo grandes sacrificios económicos para cancelar pasajes de transporte, y no es justo que un simple telefonema sea suficiente para que el médico se desligue de su responsabilidad (Colegio Médico de El Salvador, 1958: 239).

Casos como el expuesto en la cita anterior eran producto de la centralización de los servicios de salud pública hacia la mitad del siglo XX. Sólo en las principales ciudades, y excepcionalmente en ciertos municipios, contaron con algún centro de salud pública en los años cincuenta (Infante, 2000: 319-320), ya que fue en la década de los años sesenta cuando creció el número de puestos de salud (Villatoro, 1968: 27-29). Y habrá que esperar al año 1979 para que el Estado pudiera cubrir 230 municipios con alguna dependencia del Ministerio de Salud, sin garantizar la efectiva universalización de 
los servicios de salud pública (PNUD, 2010: 285-288). Hacia la primera mitad de la década de 1950, El Salvador contó con veinte centros de salud de carácter público que poseían diversos niveles de complejidad en sus servicios. Además de éstos existían cuatro hospitales especializados: psiquiátrico, militar, pediátrico y de atención a tuberculosos. En total eran veinticuatro centros asistenciales de carácter estatal (Infante, 2000: 320) para una población de 1855917 en 1950 (Digestyc, 1954: 1-3).

Los representantes estatales reconocieron las deficiencias anteriormente apuntadas e incluso las expusieron en la revista Archivos del Colegio Médico de El Salvador. Para el caso, se requería de un sistema de salud pública que funcionara como tal. Sin embargo, la primera dificultad consistía en la inexistencia de una articulación sistémica. En 1951 el Dr. Roberto Cáceres Bustamante, quien fuera subsecretario de Salud Pública y Asistencia Social, era de la opinión de que en el país no había existido un verdadero sistema de hospitales y, por lo tanto, recomendaba incidir en cuatro ejes de acción para mejorar los servicios hospitalarios: organización administrativa, preparación del personal, adquisición de equipos médicos y planificación adecuada de las instalaciones. Según Cáceres, estos cuatro aspectos abonarían para cumplir las metas a largo plazo que trazaban las consultorías internacionales, realizadas con la finalidad de mejorar los servicios de la salud pública (Cáceres, 195l: 249-255). También en 1952 el Dr. Arístides Palacios, jefe del Departamento de Medicina del hospital Rosales, consideraba que en muchos de los hospitales del país faltaba coordinación entre sus autoridades, lo que afectaba no sólo los aspectos administrativos, sino también la atención de los pacientes (Palacios, 1952: 91-92).

A propósito, en 1949, el consultor internacional Herman Grimmer, quien también publicó sus hallazgos en la revista, sostuvo que a algunos de los hospitales públicos "solamente haciendo un esfuerzo de imaginación” se les podía llamar hospitales. Algunos de ellos, visitados por Grimmer, carecían de agua y luz eléctrica, y la totalidad poseía instalaciones inadecuadas. En términos generales describió que las salas hospitalarias estaban atiborradas y sucias. Al panorama anterior habría que agregar las deficiencias en el equipo técnico para diagnosticar enfermedades y la insuficiencia de personal, en un país donde sólo siete hospitales contaban con laboratorios y únicamente seis tenían máquinas de rayos X (Grimmer, 1950). Las valoraciones de Grimmer fueron producto de un amplio análisis sobre el estado de los servicios de salud pública que presentó a las autoridades del Ministerio de Salud de El Salvador.

El ejemplo de Grimmer permite observar la atención que tuvo la salud pública en Latinoamérica por parte de organismos continentales destinados a atender temas sanitarios. Este consultor fue uno entre muchos expertos en hospitales que envió al país el Instituto de Asuntos Interamericanos (IAI), instancia del poder ejecutivo de Estados Unidos encargada de desarrollar proyectos sanitarios de manera bilateral con los Estados latinoamericanos desde 1942 (Grimmer, 1950: 199). El IAI materializó su trabajo por medio del Servicio Cooperativo Interamericano de Salud Pública, que instaló oficinas en los ministerios de salud de la región (Vieira, 1998). Esto se daba en el marco más amplio de la política exterior estadounidense del "buen vecino", que fue una respuesta diplomática para preservar la influencia hegemónica en el continente americano. Sin embargo, esta política exterior inaugurada por el presidente Franklin D. Roosevelt en 1933 tuvo variantes antes y durante la Segunda Guerra Mundial. A partir de 1941, la política del "buen vecino" tuvo como objetivo buscar alianzas en Latinoamérica, basándose en una diplomacia accesible y favorable a la cooperación económica, e incluso Estados Unidos respaldaba la ilusión de hacer creer a los estados latinoamericanos que eran iguales (Gaztambide, 2016; Hobsbawm, 2008).

A pesar de las deficiencias de los centros médicos anteriormente mencionadas, fue la DGS la instancia que abanderó un enfoque preventivo durante la década de 1950. La DGS influyó en las diferentes municipalidades del país para que acataran las disposiciones y sugerencias relativas al mejoramiento de las condiciones sanitarias, todo ello con la ayuda del Ministerio del Interior. ${ }^{5}$ La DGS pidió a las municipalidades hacer un uso correcto 
de su fondo de Higiene y Saneamiento, e invertirlo en obras que fueran estrictamente de esa naturaleza. ${ }^{6}$ Además, a los gobiernos locales se les explicó cómo implementarían las directrices de las campañas de salud preventiva que se realizaban en todo el país. ${ }^{7}$ A propósito, fue a través de las campañas antipalúdicas de la DGS ${ }^{8}$ como se logró atacar el paludismo y disminuir su influencia en los indicadores de mortalidad durante la década de 1950 (ver Cuadro 1).

Otra medida tomada en las instancias estatales fue el incremento progresivo del presupuesto destinado al sector de salud pública. Por ejemplo, en 1950 el rubro de salud tuvo una asignación presupuestaria de 2700000 colones, en 1958 recibió un monto de 17429368 colones, y en el año 1969 llegó a alcanzar la cifra de 38064627 colones (ver Cuadro 2).

Años después del periodo planteado en este artículo, en 1968 el Dr. Juan Allwood Paredes, quien se había desempeñado como director de la DGS en 1949 , sostenía que los cambios iniciados en la década de 1950 en materia de salud, no sólo en El Salvador sino en Centroamérica en general, fueron el principio de lo que él denominó la "revolución sanitaria" (Allwood, 1968: 33-35). Sin embargo, en el caso particular de El Salvador esta lectura optimista se podría circunscribir a cambios que apuntaron a la reducción del paludismo y la gastroenteritis como principales causas de muerte (ver Cuadro 1), así como a la creación del Instituto Salvadoreño del Seguro Social (ISSS) y del hospital de maternidad; también se produjeron incrementos presupuestarios en el ramo de salud pública (ver Cuadro 2) y el mejoramiento de la educación en la Facultad de Medicina de la Universidad de El Salvador, el único centro de educación superior en ese entonces.

Una lectura diametralmente opuesta a la de Allwood fue la que hizo el Dr. Carlos Alberto Villatoro, quien opinaba que en El Salvador:

La casi totalidad de la población rural no disfrutaba de los buenos servicios de la medicina científica, población que alentaba la esperanza de que, con los nuevos métodos y reformas, la escuela de medicina lograra egresar un mayor número de médicos que aliviaran, en parte, el problema de la salud (Villatoro,

1968: 23)

Entonces, es evidente que la salud pública en el periodo que se estudia presentaba un cuadro dantesco, lleno de deficiencias "trazadas en larga data" (Masferrer, 1929; Juárez 2015; Frente Democrático Estudiantil, 1944). ¿Por qué trazadas en larga data? Basta con mirar en qué se ha distribuido históricamente el dinero del Estado salvadoreño para identificar que la salud no ha sido una prioridad. Por ejemplo, después de la Independencia, a inicios del siglo XIX, los incipientes Estados de Centroamérica destinaron un aproximado del $60 \%$ de sus presupuestos para el rubro de guerra (Ramírez, 2014). Posteriormente, el Estado salvadoreño, entre 1846 y 1869 , destinó entre el 38\% y el 74\% de los recursos estatales para gastos militares y el pago de deuda (Ramírez y Walter, 2008). Para el caso, desde 1875 hasta 1900, periodo en el que se consolida el Estado salvadoreño con un poder central, el gasto militar siempre fue superior que al rubro de salud (Turcios, 2008). Luego, en el periodo comprendido entre 1897 y 1942, el Estado de El Salvador gastó en los ramos de guerra y crédito público un promedio del $46.21 \%$ de los fondos públicos (Walter, 2014); en contraste, la inversión en salud durante el periodo de 1906 a 1940 fue en promedio de un 4\% (PNUD, 2013). De seguro las cifras y datos anteriores las plasmaría años después el pintor Carlos Cañas, como acumulado del tiempo, en su poco conocida serie titulada: Hospitales, de 1973, donde se deja ver el drama social de los centros de salud salvadoreños que Cañas también vivió como paciente a mediados del siglo XX.

\section{La Universidad de El Salvador: generando opinión pública en materia de salud}

El papel de la Universidad de El Salvador en relación con la discusión sobre el tema de la salud pública durante las décadas de 1940 y 1950 pasaba por el debate interno sobre tres ideas: reforma universitaria, autonomía y función social. ${ }^{9}$ Esta situación política se abrió tras la salida del dictador Maximiliano Hernández Martínez, 
quien fue presionado por un movimiento cívico-militar en 1944 para dimitir de su cargo como presidente.

Este cambio político de 1944 propició la recuperación de la erosionada autonomía de la Universidad de El Salvador, ${ }^{10}$ lo cual contribuyó para que se discutieran temas de realidad nacional, como una posible reforma de salud y el papel social de la universidad como ente autónomo. A nivel jurídico este proceso se evidencia en la constitución de 1950 (CDJ, 1950: 25), en la nueva ley orgánica para la universidad ${ }^{11} \mathrm{y}$ en los estatutos universitarios de 1951, ${ }^{12}$ que aseguraban jurídicamente la autonomía de la Universidad de El Salvador y definieron sus funciones sociales. En este contexto la universidad fue vista por algunos de sus académicos como la orientadora del conocimiento científico que contribuiría a la solución de los problemas sociales del país, en los cuales se incluían los deficientes servicios de salud pública (Merlos, 1947). ${ }^{13}$

Ahora bien, ¿qué decían los formadores de opinión desde la Universidad de El Salvador? Por ejemplo, los estudiantes agremiados al periódico Opinión Estudiantil, en un artículo titulado: "Un girón de la tragedia médico social", publicado el 14 de junio de 1949, analizaron la situación de los servicios de salud pública y llegaron a la misma conclusión que otras voces calificadas fuera de la universidad en los siguientes términos: "Gran parte de la población urbana y rural carecen de asistencia médica... cualquier persona que visite nuestros hospitales se da cuenta de la miseria con que funcionan". ${ }^{14}$ En ese mismo artículo sostenían que ningún gobierno se había preocupado por la salud del pueblo y que se había dejado en manos incapaces la administración de los servicios públicos, por lo que demandaban a las autoridades surgidas del golpe del 14 de diciembre de 1948 que resolvieran el problema médico social. Además, cuestionaron que las instituciones de salud pública fallaran en tres aspectos: no practicaban la prevención de las enfermedades, las mujeres no recibían adecuada atención prenatal y los trabajadores de la salud realizaban sus tareas con presupuestos escasos, lo que repercutía en la calidad de los servicios. ${ }^{15}$

Hacia el año 1950, Opinión Estudiantil ya había endurecido su línea editorial y se tornó en un acérrimo crítico del movimiento político de 1948, que había apoyado en sus primeros meses. Al reflexionar sobre la problemática de la salud mencionaron comentarios como los siguientes contra los revolucionarios de diciembre de 1948: “[...] mientras se compra un millón de colones en armas a los Estados Unidos, los enfermos del hospital Rosales duermen en el suelo y los centros hospitalarios están casi en la ruina total". ${ }^{16}$

Pero los estudiantes de Opinión Estudiantil no adjudicaban toda la responsabilidad al Estado, pues exigían al sector empresarial contribuir en la mejora de la salud de aquel pueblo que forjaba sus capitales. ${ }^{17}$ Y esto tenía sentido en un país en el que en 1950 se calculaba que había invertido 245000000 de colones en el bosque cafetero nacional, mientras la población apenas recibió 17160000 colones en concepto de atención en salud (Tejeda, 1950: 275-276). En otras palabras, estos universitarios tenían un planteamiento redistributivo de la riqueza frente a aquella economía cafetalera que generaba una alta acumulación de capital y una disminuida distribución de la riqueza que afectaba el rubro de la salud.

Fue en este panorama como los servicios sociales que podían desarrollar los estudiantes universitarios formaban parte de esa concreción de la función social de la universidad, a la que apelaban los académicos que opinaban sobre la salud pública en El Salvador. Por razones como ésta, los estudiantes de medicina se consideraban necesarios en un país desprovisto de asistencia sanitaria oportuna y carente de la lógica de sistema. Verbigracia, un artículo titulado: "Lo que queremos de nuestra universidad", publicado en otro periódico universitario llamado Líder, se expresó al respecto: "Nosotros llevaremos al campo la mano servicial y bondadosa del médico y del estudiante de medicina, logrando combatir las enfermedades que minan la potencia trabajadora de nuestra clase campesina" (Frente Democrático Estudiantil, 1944: 3). Esta última cita es un ejemplo claro de la medicalización militante de la que hablaba Foucault.

En una publicación de 1947 de la revista La Universidad, el académico Salvador Merlos consideró que la Universidad de El Salvador, por su carácter 
público, tenía como objetivo retribuir a la sociedad salvadoreña por medio de sus diferentes disciplinas. Merlos opinó que la función social de la universidad debía ir al encuentro de las necesidades de la sociedad e inculcar en los estudiantes un espíritu de servicio (Merlos, 1947: 191-193). Al referirse en particular a la labor sanitaria que podía desarrollar la universidad, lo acotó así:

De gran utilidad sería la divulgación de conocimientos sanitarios mediante sistemas de conferencias y cartillas puestas al alcance de nuestro pueblo; ella [la universidad] tiene los técnicos de ese importante ramo y le es fácil abordar y divulgar conocimientos sobre paludismo, enfermedades venéreas, alcoholismo, nutrición, parásitos intestinales, etc... La universidad debe salir de su recinto y llegar al taller y a la aldea lejana llevando salud [...] (Merlos, 1947: 192).

En sintonía con lo anterior, en julio de 1949 el rector de la Universidad de El Salvador manifestó en la sesión del Consejo Superior Directivo que, de acuerdo con los estudiantes de medicina y odontología, se deseaba establecer el servicio social obligatorio de seis meses. Se agregaba que las autoridades de las facultades de medicina y odontología debían establecer comunicación con la entonces instancia de Salud Pública y Asistencia Social ${ }^{18}$ con la finalidad de concretar los servicios sociales obligatorios de sus alumnos debidamente reglamentados. ${ }^{19}$ Incluso, se planteó hacer extensivo el servicio social a las demás facultades y para ello se pedía un plan de trabajo que debía quedar sujeto a la aprobación del Consejo Superior Directivo. La necesidad de médicos y odontólogos para servicio social era evidente, al grado que el ramo de la salud pública o la misma universidad flexibilizaban los requisitos de los reglamentos que regían a las facultades de medicina y odontología. ${ }^{20}$

Seguramente, el nivel de la discusión en torno a la salud pública en El Salvador fue uno de los catalizadores para elevar la calidad educativa de la Facultad de
Medicina de la Universidad de El Salvador a finales de la década de 1950. Esta misión para la mejora educativa fue realizada por un equipo de médicos que se habían especializado en México y Estados Unidos y tenía como líder al Dr. Fabio Castillo Figueroa. Tan responsable fue el proyecto de Castillo, que la Facultad de Medicina tenía un exigente proceso de selección de aspirantes, al grado que esto fue causa de conflictos con las autoridades universitarias. A propósito, en 1960 la Fundación Rockefeller opinaba de la siguiente manera sobre la Facultad de Medicina de la universidad: "[...] esta escuela puede convertirse en un centro que se considere líder para otros países de Centroamérica con respecto a estándares más altos en educación [...] gracias a la inspiración de gente como Fabio Castillo" (Lindo, 2012: 1).

En 1957, la Universidad de El Salvador por medio de su Facultad de Medicina institucionalizó la cátedra de medicina preventiva e higiene. Seguramente este hecho respondía a la discusión que se vivió en El Salvador con respecto a la creación de un sistema de salud pública de impacto nacional durante esa década de 1950 y al fortalecimiento que tuvo la misma facultad en ese contexto (Villatoro, 1968: 25).

\section{Conclusión}

Las opiniones expuestas por los médicos y los universitarios salvadoreños refuerzan la aseveración foucaultiana que concibe a los emisarios de la salud y sus conocimientos como actores esenciales que influyen en la sociedad, bajo el paradigma del cuerpo sano y de que la sociedad necesita ser corregida de sus males. Esto admite asegurar que se produjo una narrativa médica que amoldó la opinión pública en El Salvador, sobre todo en aquellos estratos interesados en los problemas de los servicios de salud pública.

Lo expuesto permite plantear la hipótesis siguiente: fue a partir de la década de 1950 cuando el Estado pensó la salud pública bajo la lógica de un sistema de impacto nacional. 


\section{Notas}

1 Jorge Juárez (2015) y Diana Durán (2016) son los únicos autores que desde la historiografía salvadoreña se han interesado por la salud pública como problema de investigación.

${ }^{2}$ Las nociones de opinión pública que más han influido son las resultantes de la modernidad, que están íntimamente vinculadas al desarrollo de las ideas de soberanía popular, formas de gobierno y autonomía individual (Monzón, 1985: 81-109). En este texto se entiende como opinión pública el conjunto de información que configura consensos discursivos que son producidos en instancias sociales que disciplinan la visión de la realidad. Lo anterior no está exento de discursos subalternos que están contrapuestos al consenso discursivo en su relación dialéctica. Particularmente, el conocimiento médico disciplina la opinión pública bajo la siguiente premisa: la sociedad está necesitada de conocimiento médico para alcanzar de nuevo el estado de buena salud del cuerpo social; a esto último Michel Foucault (2004: 57) lo categorizó como "medicalización dogmática" de la sociedad.

3 Estos médicos comprendían la medicina social como la destinada a intervenir en las condiciones medioambientales que afectan la salubridad de la sociedad, siendoel Estadoel garante del derecho ala salud. Hay que aclarar que a finales de los años sesenta del siglo pasado la medicina social en Latinoamérica se radicalizó hacia posturas más críticas del orden económico, con una clara influencia marxista. Para profundizar en este tema ver Iriart et al. (2002: 128-134).

${ }^{4}$ Se trata de un suceso político conducido por un grupo de militares jóvenes con el respaldo de un grupo de líderes civiles que proclamaron iniciar una revolución. Los cambios tuvieron el tinte de la modernidad y del autoritarismo, pero no se puede negar que cambiaron la concepción del Estado salvadoreño, por uno de cuño más intervencionista en lo social y económico. Ello se tradujo gradualmente en un cambio en la estrategia de desarrollo que aspiraba a consolidar un Estado social. Todo esto en un contexto de bonanza del monocultivo del café y una timorata industrialización del país.
${ }^{5}$ Para sustentar esta parte se recurrió a correspondencia y documentos diversos. AGN (Archivo Nacional de la Nación) (1952-1955). Fondo Ministerio del Interior, series Dirección General de Sanidad y Ministerio de Salud, caja 2 y 3. San Salvador: Carta del Ministerio de Salud al Ministerio de Interior, 1952; nota de la DGS, 1952; Circular 166, 1952; Carta del Ministerio del Interior, 1952; circular 38, 1952.

${ }^{6}$ Informes de la División de Ingeniería Sanitaria (1950). [Informe]. AGN, fondo gobernación política de Nueva San Salvador, serie documentos buenos, caja 17. San Salvador; Circular 141 (1952). [Carta]. AGN, Fondo Ministerio del Interior, serie Dirección General de Sanidad, caja 2. San Salvador.

${ }^{7}$ Para sustentar esta parte se recurrió a correspondencia y documentos diversos. AGN (Archivo Nacional de la Nación) (1952-1955). Fondo Ministerio del Interior, series Dirección General de Sanidad y Ministerio de Salud, caja 2 y 3. San Salvador. A continuación, el detalle por documentos: Esquema nacional de la campaña contra las moscas, 1952; notas núm. 2828, 3216, 3217 y 19796 de 1952; sinopsis de la reunión en Sonsonate, 1952. Esta reunión en Sonsonate trató sobre los aspectos de cómo los municipios del departamento de Sonsonate aplicarían las directrices de las autoridades sanitarias de El Salvador.

8 "Memorándum ampliación de la campaña nacional anti-palúdica tendiente a erradicar la enfermedad en El Salvador" (1955). [Informe]. San Salvador: AGN, Fondo Ministerio del Interior, serie ministerio de salud, caja 3. San Salvador.

${ }^{9}$ Parallegar a la conclusión de que estos fueron los temas de discusión al interior de la Universidad de El Salvador se utilizó el siguiente dossier de fuentes del periódico Opinión Estudiantil: Estudiantes de la Universidad de El Salvador (en adelante EUES) (27 de mayo de 1944), "Función social de la universidad". Opinión Estudiantil, 1 y 3; EUES (14 de enero de 1949). "La autonomía universitaria". Opinión Estudiantil, 1 y 4; EUES (4 de mayo de 1949). "Sobre la reforma universitaria". Opinión Estudiantil, 1 y 4; EUES (6 de junio de 1949). "Con los nuevos directivos estudiantiles". Opinión Estudiantil, 1-2; EUES (14 de junio de 1949). "Un girón de la tragedia médico-social". Opinión 
Estudiantil, 1-2; EUES (18 de junio de 1949). "El momento político actual y la unidad estudiantil”. Opinión Estudiantil, 1-2; EUES (2 de julio de 1949). "Hacia una cultura verdaderamente popular". Opinión Estudiantil, 1-2; EUES (22 de agosto de 1949). "Nuestra universidad y su misión cultural”. Opinión Estudiantil, l; EUES (2 de junio de 1950). "Docencia universitaria". Opinión Estudiantil, 2; EUES (23 de junio de 1950). "Participación del estudiante en el gobierno universitario". Opinión Estudiantil, 2-3; EUES (7 de julio de 1950). "El pueblo, los estudiantes y nuestra falsa revolución”. Opinión Estudiantil, 1 y 4; EUES (25 de julio de 1950). "Editorial. Misión universitaria y por la reforma”. Opinión Estudiantil, 1-2; EUES (2 de agosto de 1950). "Carta abierta al consejo superior directivo de la Universidad". Opinión Estudiantil, 2; EUES (4 de agosto de 1950). "Los ideales porque luchamos". Opinión Estudiantil, 3; EUES (16 de agosto de 1950). "Por una universidad al servicio de las mayorías". Opinión Estudiantil, 1 y 4; EUES (2 de septiembre de 1950). "Por la reforma universitaria". Opinión Estudiantil, 1-4; EUES (3 de octubre de 1950). "Editorial y las enmiendas a los estatutos universitarios". Opinión Estudiantil, 1.

${ }^{10}$ Decreto 44 (28 de agosto de 1940). Diario Oficial, 2769 2770; Decreto 13 (29 de julio de 1944). Diario Oficial, 2314.

${ }^{11}$ Decreto 122, Ley Orgánica de la Universidad de El Salvador (31 de enero de 1951). Diario Oficial, 471-473.

12 Acuerdo de Estatuto Orgánico (3 de septiembre de 1951). Diario Oficial, 5309-5323.

${ }^{13}$ Ver también: EUES (27 de mayo de 1944). "Función social de la universidad”. Opinión Estudiantil, 1 y 3; EUES (6 de junio de 1949). "Con los nuevos directivos estudiantiles”. Opinión Estudiantil, 1-2; EUES (3 de octubre de 1950). "Editorial y las enmiendas a los estatutos universitarios". Opinión Estudiantil, 1.

${ }^{14}$ EUES (14 de junio de 1949). "Un girón de la tragedia médico-social”. Opinión Estudiantil, 1-2.

${ }^{15}$ EUES (14 de junio de 1949). "Un girón de la tragedia médico-social”. Opinión Estudiantil, 2.

${ }^{16}$ EUES (7 de julio de 1950). "El pueblo, los estudiantes y nuestra falsa revolución”. Opinión estudiantil, 4.

${ }^{17}$ EUES (14 de junio de 1949). "Un girón de la tragedia médico-social”. Opinión Estudiantil, 2.
${ }^{18}$ Universidad de El Salvador (7 de julio 1949). Actas de sesiones de los consejos: superior directivo y ejecutivo. San Salvador, 171 y 175 .

${ }^{19}$ Universidad de El Salvador (6 de octubre de 1949). Actas de sesiones de los consejos: superior directivo y ejecutivo. San Salvador, 220 y 224-225.

${ }^{20}$ Universidad de El Salvador (3 de febrero de 1950). Actas de sesiones de los consejos: superior directivo y ejecutivo. San Salvador: Universidad de El Salvador, 132 y 135 136; Universidad de El Salvador (13 de febrero de 1950). Actas de sesiones de los consejos: superior directivo y ejecutivo. San Salvador, 20 y 22-23. Estas actas abordan los aspectos administrativos, políticos y académicos de la Universidad de El Salvador. En ellas es posible identificar la toma de decisiones como cuerpo colegiado que conducía a la universidad. Para el presente trabajo se prestó especial atención a lo relacionado con la función social y los servicios sociales de medicina.

\section{Referencias}

Allwood Paredes, Juan (1949). "Breve reseña de las investigaciones de bacteriología intestinal en El Salvador". En Archivos del Colegio Médico de El Salvador, 2(2): 106.

Allwood Paredes, Juan (1968). Los recursos de la saludpública en Centroamérica. San Salvador: Secretaría General de la Organización de Estados Centroamericanos.

Cáceres Bustamante, Roberto (1951). "Plan hospitalario de El Salvador". En Archivos del Colegio Médico de El Salvador, 4: 249-255.

Calderón, Roberto (1949). "Cardiopatología postmortem". En Archivos del Colegio Médico de El Salvador, 2(2): $73-74$.

CDJ (Centro de Documentación Judicial) (1950). "Constitución de El Salvador 1950". Disponibleen:http:// www.jurisprudencia.gob.sv/DocumentosBoveda/ D/2/1950-1959/1950/09/886F2.PDF.

Colegio Médico de El Salvador (1949a). "La medicina y la nueva constitución política". En Archivos del Colegio Médico de El Salvador, 2(2): 133.

Colegio Médico de El Salvador (1949b). "Reseña histórica de los congresos médicos en El Salvador”. En Archivos del Colegio Médico de El Salvador, 2(4): 268. 
Colegio Médico de El Salvador (1950). "La organización técnica de los servicios médicos". En Archivos del Colegio Médico de El Salvador, 3(1): 51.

Colegio Médico de El Salvador (1958). Función del médico en las consultas externas. Archivos del Colegio Médico de El Salvador, 11 (4), 239.

DIGESTyC (Dirección General de Estadística y Censos) (1954). Segundo censo de población 1950. San Salvador: DIGESTyC

Durán Marroquín, Diana Carolina (2016). Historia de las políticas públicas de salud sobre las enfermedades transmisibles enEl Salvador 1950-2000. Tesis de pregrado, Universidad de El Salvador, San Salvador, El Salvador.

Foucault, Michel (1978). "Incorporación del hospital en la tecnología moderna”. En Educación Médica y Salud, 12(1): 20-33.

Foucault, Michel (2004). El nacimiento de la clínica una arqueología de la mirada médica. Avellaneda, Argentina: Siglo XX Editores.

Frente Democrático Estudiantil (1944). "Lo que queremos de nuestra universidad". En Líder, 3, 18 de octubre.

Gaztambide, Antonio (2016). "Buena vecindad y populismo: el caribe en las relaciones interamericanas de 1936 a 1946". En Estudios e Pesquisas sobre as Américas, 2: 4-9.

Grimmer, Herman (1950). "Programa nacional de hospitales generales en El Salvador". En Archivos del Colegio Médico de El Salvador, 3(4): 199-226.

Hobsbawn, Eric (2008). Historia del siglo XX. Barcelona: Crítica.

Infante Meyer, Carlos (2000). Historia de la medicina en El Salvador. San Salvador: Ancalmo Internacional.

Iriart, Celiaet al.(2002). "Medicina sociallatinoamericana: aportes y desafíos". En Revista Panamericana de Salud Pública, 12(2): 128-134.

Juárez, Jorge (2015). Población: estadísticas y enfermedades en la configuración del Estado en El Salvador en la segunda mitad del siglo XIX [documento inédito]. El Salvador: Instituto de Estudios históricos, Antropológicos y Arqueológicos, Universidad de El Salvador.

Lima, Ernesto R. (1951). Informe de la labor desarrollada como médico rural en la ciudad de Jayaque departamento de La Libertad del 1 de enero al 30 de junio de 195l. Tesis doctoral, Universidad de El Salvador, San Salvador.

Lindo, Héctor (2012). "Lecciones de liderazgo de Fabio Castillo Figueroa”. EnElFaro, 4 denoviembre. Disponible en: https:/elfaro.net/es/201211/opinion/10122/.

López Bernal, Carlos Gregorio (2000). “Años de bonanza y crisis; de ilusiones y desencantos”. En Álvaro Magaña (ed.), El Salvador; la república. San Salvador: Fomento Cultural, Banco Agrícola, pp. 374-376.

Merlos, Salvador (1947). "Visión panorámica de la reforma Universitaria”. En La Universidad, 1: 187 y 191-194.

Masferrer, Alberto (1929). El mínimum vital. San Salvador: Editorial Helios.

Ministerio de Economía. Dirección General de Estadísticas y Censos (1958). Hechos y cifras de El Salvador 1957. San Salvador: Dirección General de Estadísticas y Censos.

Ministerio de Economía. Dirección General de Estadísticas y Censos (1964). Hechos y cifras de El Salvador 1964. San Salvador: Dirección General de Estadísticas y Censos.

Monzón, Candido (1985). Orígenes y primeras teorías sobre la opinión pública: El liberalismo y el marxismo". Estudios políticos, 44, 81-109.

Oliva Mancia, Mario Daniel Ernesto (2011). Ciudadanía e higienismo social en El Salvador 1880-1932. Tesis doctoral, Universidad Centroamericana, San Salvador.

Pacheco Araujo, José (1949). "Descripción de los servicios médicos rurales: sugerencia sobre su posible implantación en El Salvador". En Archivos del Colegio Médico de El Salvador, 2(4): 219-242.

Palacios, Arístides (1952). "¿Pueden nuestros hospitales rendir más?” En Archivos del Colegio Médico de El Salvador, 5(2): 91-92.

Peñalver, Luís et al. (1956). "El diagnóstico del paludismo en el medio hospitalario”. En Archivos del Colegio Médico de El Salvador, 9(1): 1 .

PNUD (Programa de las Naciones Unidas para el Desarrollo) (2010). Informe sobre desarrollo humano El Salvador 2010. De la pobreza y el consumismo al bienestar de la gente. Propuestas para un nuevo modelo de desarrollo. San Salvador: PNUD. 
PNUD (Programa de las Naciones Unidas para el Desarrollo) (2013). Informe sobre desarrollo humano El Salvador 2013. Imaginar un nuevo país. Hacerlo posible. Diagnóstico y propuesta. San Salvador: PNUD.

Quinteros, Edgar y Roberto Mejía (2018). "Calidad microbiológica de agua envasada en El Salvador 2014 2015". En Alerta, 1: 26-34.

Ramírez, José Alfredo y Knut Walter (2008). "Los estancos en la república del Salvador 1838-1871". En Los estancos las prácticas monopólicas y las rentas del estado en El Salvado. San Salvador: DPI Concultura, Superintendencia de Competencia, pp. 151-155.

Ramírez, Sigfredo (2014). "Nos duele ser tan pequeños". En La Prensa Gráfica, 21 de septiembre. Disponible en: https:/www.laprensagrafica.com/revistas/Nosduele-ser-tan-pequenos-20140921-0020.html

Rojas Bolaños, Manuel (1993). "La política". En Héctor Pérez Brignoli (ed.), Historia general de Centroamérica de la posguerra a la crisis1945-1979. Madrid: Siruela.

Tejeda Paris, Enrique (1950). "Plan de investigaciones gubernamentales para la República de El Salvador". En Revista de Economía de El Salvador, 1-4: 275-276.
Turcios, Roberto (1993). Autoritarismo y modernización. San Salvador: Ediciones Tendencias.

Turcios, Roberto (2003). Autoritarismo y modernización. San Salvador: DPI Concultura.

Turcios, Roberto (2008). "Paisaje de reformas 1871-1897". En Los estancos las prácticas monopólicas y las rentas del estado en El Salvado. San Salvador: DPI Concultura, Superintendencia de Competencia, p 216-217.

Vieira, André Luiz (1998). "The Institute of InterAmerican Affairs and its Health Policies in Brazil during World War II". En Presidential Studies Quarterly, 28: 523-526.

Villatoro, Carlos Alberto (1968). Breve estudio de la medicina rural en El Salvador. Tesis doctoral, Universidad de El Salvador, San Salvador.

Walter, Knut (2014). Las políticas culturales del Estado salvadoreño 1900-2012. San Salvador: Fundación Acces Arte.

Zelaya, Susana y Roberto Mejía (2018). "Mortalidad y años de vida potencialmente perdidos por enfermedades no transmisibles en El Salvador, 20112015". En Alerta, 1: 44-52. 


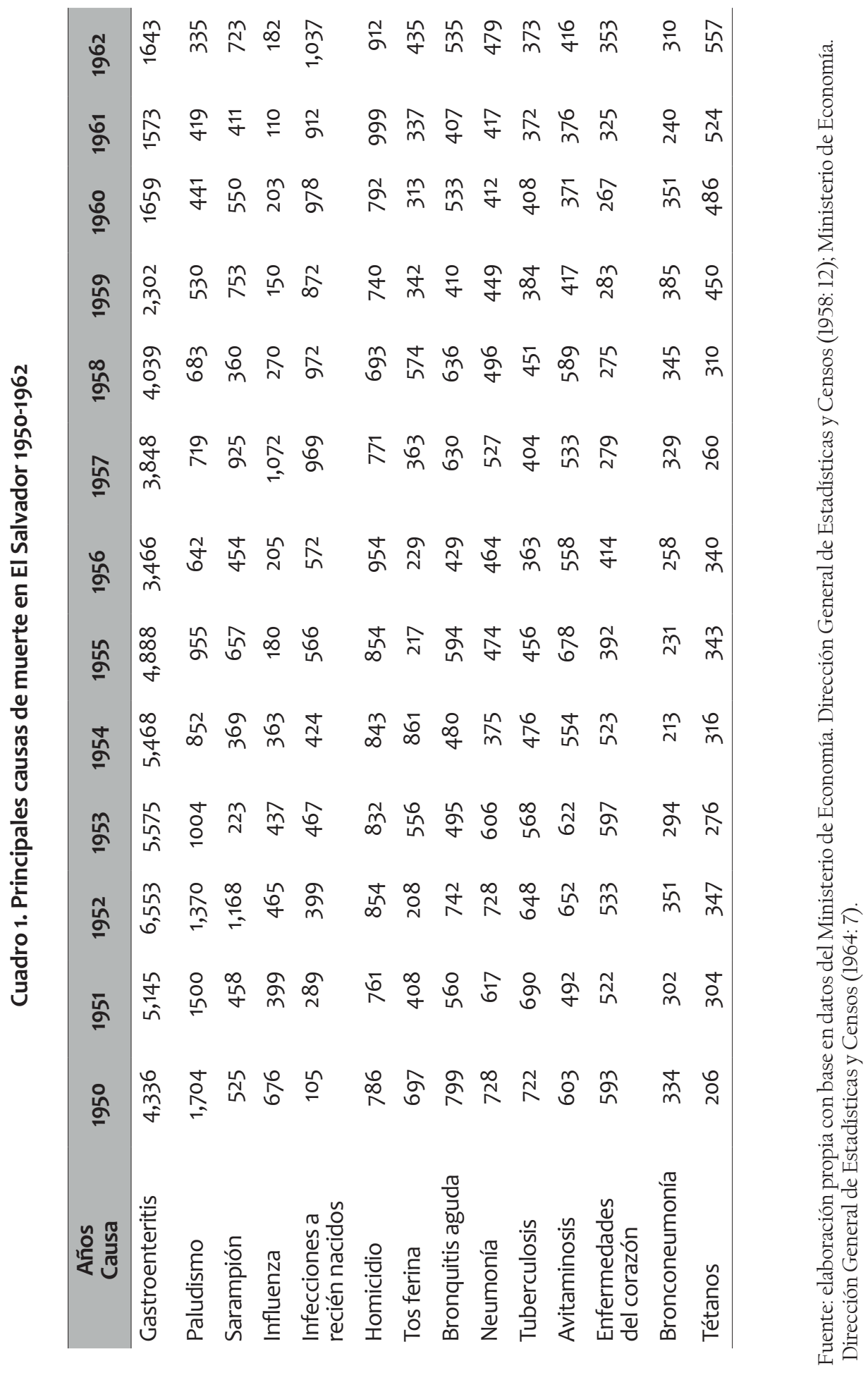




\section{Cuadro 2. Montos presupuestarios del sector de salud pública 1950-1968}

\begin{tabular}{|c|c|}
\hline AÑos & MONTO EN COLONES \\
\hline 1950 & $2,700,000$ \\
\hline 1951 & $11,970,000$ \\
\hline 1952 & $13,073,485$ \\
\hline 1953 & $13,350,394$ \\
\hline 1954 & $14,619,680$ \\
\hline 1955 & $16,155,000$ \\
\hline 1956 & $15,200,000$ \\
\hline 1957 & $11,834,186$ \\
\hline 1958 & $17,429,368$ \\
\hline 1959 & $18,054,747$ \\
\hline 1960 & No se encontró \\
\hline 1961 & $19,450,793$ \\
\hline 1962 & $20,299,867$ \\
\hline 1963 & $21,004,717$ \\
\hline 1964 & $22,318,700$ \\
\hline 1965 & $21,517,460$ \\
\hline 1966 & $29,632,136$ \\
\hline 1967 & $32,141,484$ \\
\hline 1968 & $35,642,583$ \\
\hline 1969 & $38,064,627$ \\
\hline
\end{tabular}

Fuente: elaboración propia con base en Ministerio de Economía. Dirección General de Estadísticas y Censos (1958: 35) y Walter (2014: 92-93). 
Foto 1. Pacientes en clínica asistencial de Jayaque, 1951

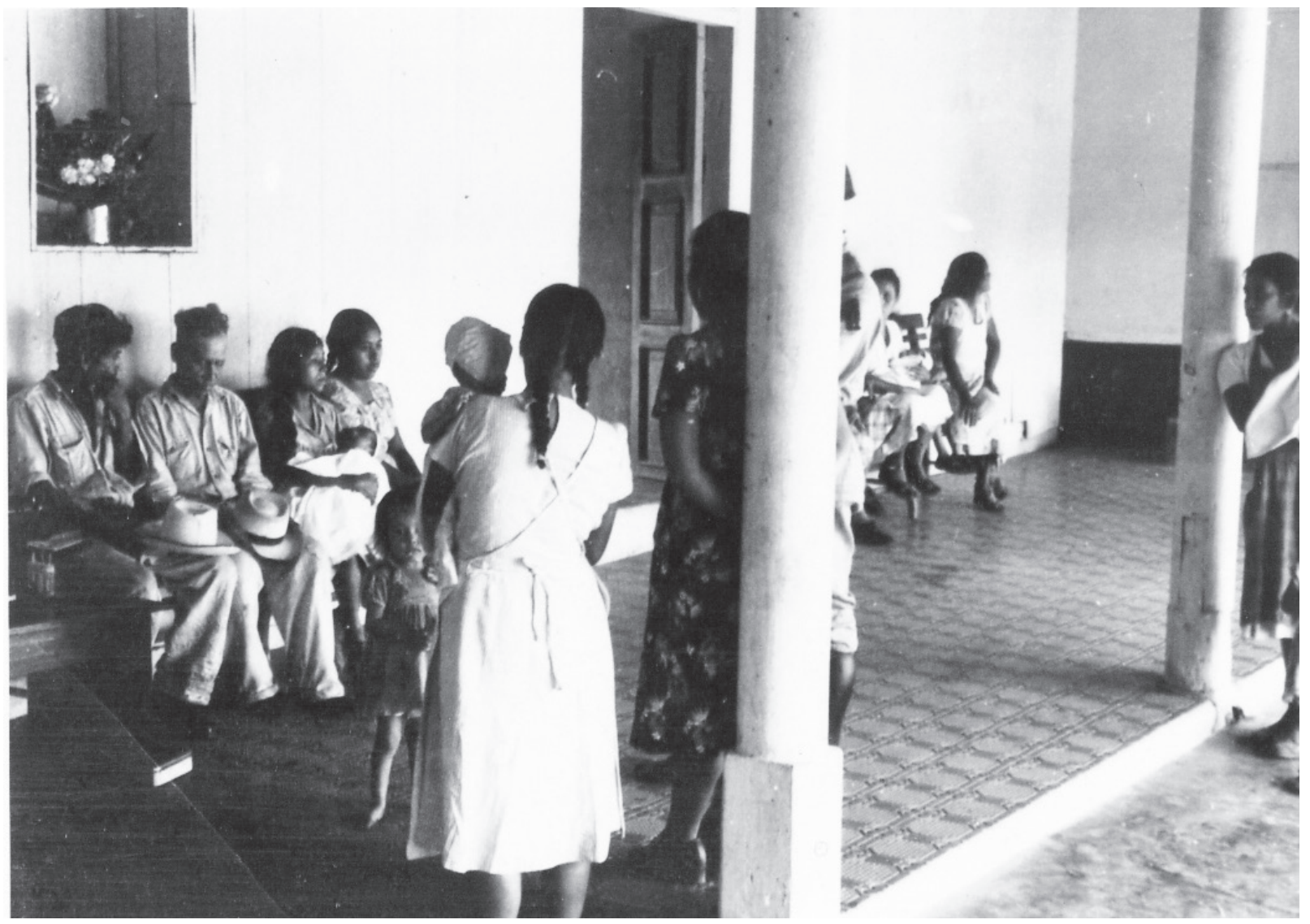

Fuente: Lima, Ernesto R. (1951). "Informe de la labor desarrollada como médico rural en la ciudad de Jayaque departamento de La Libertad, del 1 de enero al 30 de junio de 1951”. Tesis doctoral, Universidad de El Salvador, San Salvador. 
Foto 2. Portada de la revista Archivos del Colegio Médico de El Salvador, junio de 1956

\section{ARCHIVOS}

DEL

\section{COLEGIO MEDICO DE EL SALVADOR}

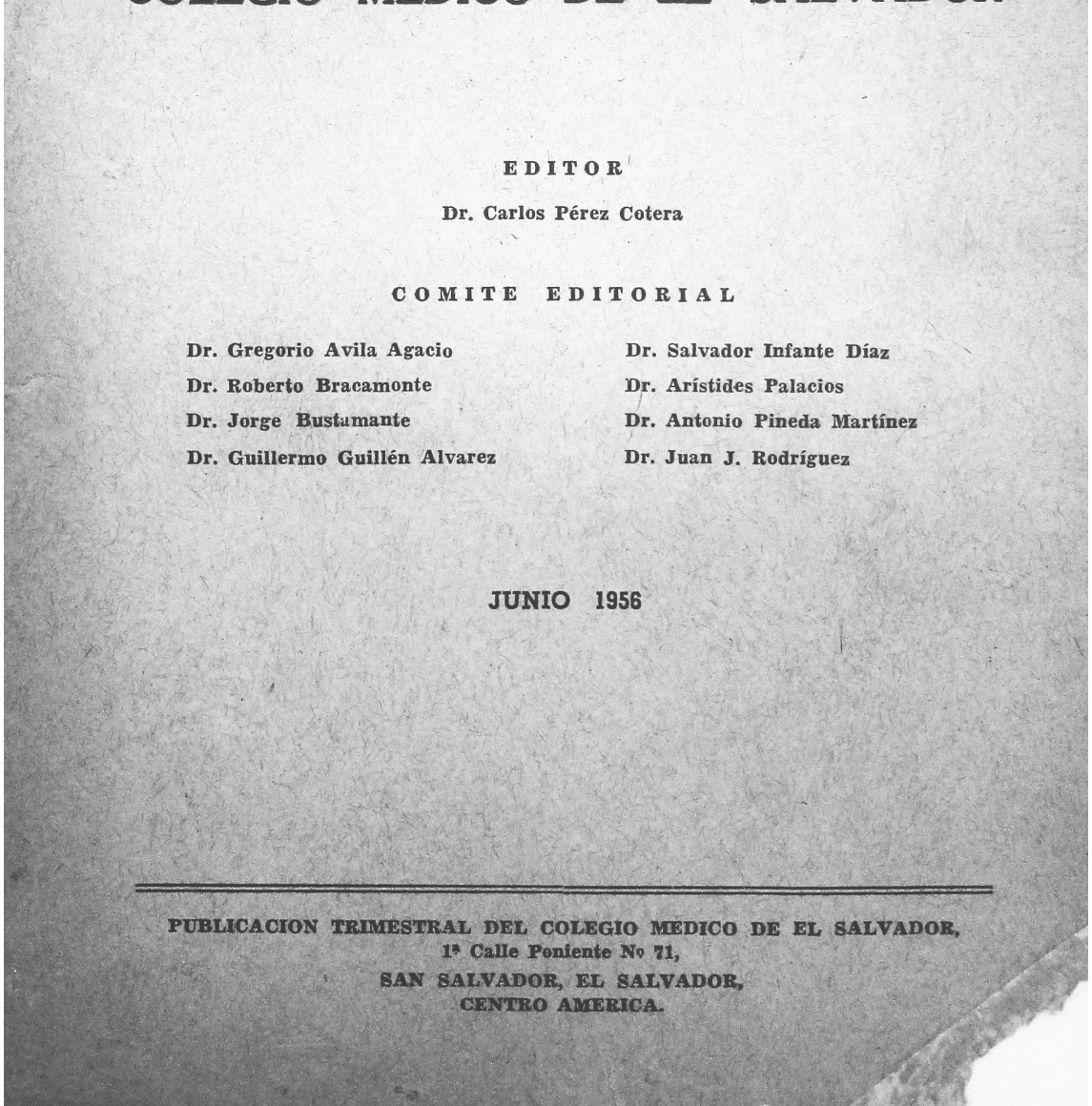

Fuente: Colegio Médico de El Salvador. 